\title{
635 ONLINE CME IMPROVES THE INTERPROFESSIONAL TEAM'S ABILITY TO MANAGE PATIENTS WITH MALIGNANT PLEURAL MESOTHELIOMA
}

${ }^{1}$ Michelle Worst*, ${ }^{*}$ Emily Van Laar, ${ }^{1}$ Megan Whitney, ${ }^{2}$ Anne Tsao, ${ }^{3}$ Hossein Borghaei. ${ }^{1}$ Medscape Oncology, New York, NY, United States; ${ }^{2}$ University of Texas MD Anderson Cancer Center, Houston, TX, United States; ${ }^{3}$ Fox Chase Cancer Center, Philadelphia, PA United States

Background Malignant pleural mesothelioma (MPM) is a rare and aggressive inflammatory cancer commonly associated with prior exposure to asbestos. Until recently, approved systemic treatments for MPM have been limited to chemotherapy regimens that have had limited survival benefit with overall poor outcomes. Both immune checkpoint inhibitors (ICIs) and tumor treating fields (TTF) have been associated with improved survival in this population. As a result of these advancements and changing of the treatment landscape, many members of the interprofessional team are challenged to stay current with emerging data and knowing how best to integrate these regimens into MPM treatment paradigms. The objective of this study was to determine if an online continuing medical education (CME) intervention could improve oncologists' and pharmacists' ability in managing patients with MPM.

Methods The activity consisted of a 30-minute video discussion between two expert faculty. ${ }^{1}$ Educational effect was assessed with a repeated pairs pre-/post-assessment study including a 3-item, multiple choice, knowledge/competence questionnaire and one confidence assessment question, with each participant serving as his/her own control. Pre- and postassessment scores were compared to determine relative changes in the proportion of correct responses to knowledge/ competence questions. A paired samples t-test assessed overall number correct and confidence change statistical significance . The activity launched 5 January 2021; data were collected until 3 May 2021.

Results Overall, statistically significant improvements in knowledge/competence were seen after education consumption for oncologists $(\mathrm{N}=44, \mathrm{P}<.001)$ and pharmacists $(\mathrm{N}=223$, $\mathrm{P}<.001)$. Relative improvement in correct answers was $79 \%$ for oncologists and $123 \%$ for pharmacists (total correct responses pre-/post-assessment were $33 \% / 59 \%$ and 22\%/49\%, respectively). Following the activity, $41 \%$ of oncologists and $57 \%$ of pharmacists had a measurable increase in confidence regarding their ability to collaborate as part of the interprofessional team in the management of patients with MPM.

Conclusions Participation in an online, video discussion-based CME-certified activity resulted in statistically significant improvements in knowledge/competence, and measurable increases in confidence of oncologists and pharmacists regarding the management of patients MPM. These results have the ability to translate to improvements in clinical care. The need for additional educational activities was also identified to address residual gaps and further increase clinicians' ability in this clinical setting.

Acknowledgements Sources of support: Developed through an independent educational grant from Bristol Myers Squibb Company and Novocure.

\section{REFERENCE}

1. Tsao A, Borghaei H. Moving the Needle in Mesothelioma: advances in treatment. Medscape 2021. https://www.medscape.org/viewarticle/943353. 